\section{Reactive Hypoglycemia From Metformin Immediate-Release Monotherapy Resolved by a Switch to Metformin Extended-Release: Conceptualizing Their Concentration-Time} Curves

\author{
Ayesha Akram 1,2 \\ 1. Internal Medicine, Combined Military Hospital, Rawalpindi, PAK 2. Internal Medicine, Rawalpindi Medical \\ University, Rawalpindi, PAK
}

Corresponding author: Ayesha Akram, ayesha_akram_01@hotmail.com
Review began 06/10/2021 Review ended 06/21/2021 Published 07/02/2021

๑) Copyright 2021 Akram. This is an open access article distributed under the terms of the Creative Commons Attribution License CC-BY 4.0., which permits unrestricted use, distribution, and reproduction in any medium, provided the original author and source are credited.

\begin{abstract}
Metformin rarely, if ever, causes hypoglycemia when it is used as labeled. A 55-year-old woman presented to the medicine ward with an altered level of consciousness. She had been reviewed in an outpatient department three days earlier and prescribed $500 \mathrm{mg}$ two times per day of metformin immediate-release (Met IR) for newly diagnosed type 2 diabetes mellitus (T2DM), to which she had been adherent; however, she had been experiencing intermittent episodes of hypoglycemia after taking the medication prescribed to treat her T2DM. On physical examination, she was diaphoretic and disoriented but responsive to sensory stimuli. In the ward, she received $25 \mathrm{ml}$ of intravenous dextrose as the initial blood glucose reading was low at 54 $\mathrm{mg} / \mathrm{dl}$, and 4 ounces of apple juice additionally two hours later as her blood glucose level fell below $70 \mathrm{mg} / \mathrm{dl}$ again. She was no longer hypoglycemic a few hours later, and there was a significant neurological improvement. The remainder of the laboratory results, including serum renal and liver function tests, were normal. Met IR was discontinued, and metformin extended-release (Met XR) $500 \mathrm{mg}$ /day was initiated at discharge. The patient's hypoglycemic episodes resolved within days after the initiation of Met XR. Hypoglycemia is rarely associated with accidental or suicidal overdose of metformin, metabolic dysfunction (e.g., renal insufficiency), exercise, missed meal, acute illness, or the initiation of additional antidiabetic medication. Albeit even uncommon, metformin-associated hypoglycemia may occur with no obvious trigger. In this context, we determine to what extent Met IR may contribute to the development of hypoglycemia in an individual case, but also that the risk could be mitigated by a switch to Met XR. In a preferred embodiment, the Met XR dosage form can be administered once a day, ideally with or after a meal, preferably with or after the evening meal, and it provides therapeutic levels of the drug throughout the day with peak plasma levels being obtained between four to eight hours after the administration $\left(\mathrm{T}_{\max }\right)$.
\end{abstract}

Categories: Endocrinology/Diabetes/Metabolism, Internal Medicine

Keywords: glycemic control, metformin immediate release, metformin extended release, therapeutic indications, adverse reactions, hypoglycemia

\section{Introduction}

The term antidiabetic or antihyperglycemic drugs refers to drugs that are useful in managing type 2 diabetes mellitus (T2DM). Preferably, a first-line antidiabetic drug is a biguanide such as metformin or its pharmaceutically acceptable salt such as metformin hydrochloride. Metformin is an oral antihyperglycemic drug that improves glucose tolerance in T2DM by lowering both basal and postprandial plasma glucose [1]. Metformin exerts its metabolic effects by reducing hepatic gluconeogenesis due to the inhibition of mitochondrial glycerophosphate dehydrogenase, decreases hepatic lipogenesis by upregulation of AMPactivated protein kinase, and increases insulin-dependent peripheral glucose uptake and utilization [1,2]. In addition, it reduces appetite and decreases intestinal absorption of glucose [1]. The dosage of metformin is individualized on the basis of both effectiveness and tolerance in adults [1].

Previously, metformin was available as an immediate-release formulation (Met IR) only. Any such formulation is quickly absorbed and then rapidly cleared, necessitating multiple dosing with considerable plasma level fluctuations consisting of peaks and troughs. These fluctuations may account for transient side effects at the peak and a potential fall to subtherapeutic levels at the trough. This ultimately is the rationale behind developing a newer formulation, namely, metformin extended-release (Met XR) [3-5]. Met XR tablets are designed with the active ingredient overlaid with an enteric coat. The active drug is released through hydrated polymers, which expand the safe uptake of fluid, thereby prolonging gastric transit and delaying drug absorption in the upper gastrointestinal tract [1]. Owing to its slower absorption, Met XR can provide non-pulsating, therapeutic levels over a 12- to 24-hour period with less frequent administration, typically once daily [1]. Tolerability and even glycemic control in T2DM have been reported to improve with Met XR. Patient satisfaction and compliance are increased using a reduced dosing interval at one versus two or three 
times daily [3-5]. The bioavailability of the drug is not decreased by the presence of food, in fact a slight increase in the bioavailability is observed when the Met XR dosage form is administered with food [1]. Circulating levels of endogenous insulin are unchanged or slightly decreased by Met XR, and hence it carries a low risk of hypoglycemia [1].

Adverse events associated with metformin use are often gastrointestinal in nature, e.g., nausea, vomiting, and occasionally diarrhea [1]. However, hypoglycemia due to overdose, decreased drug clearance, nutritional deficits, multiple comorbidities, and drug-drug interactions, is rarely known to occur [6-11]. In this report, the case of a 55-year-old diabetic patient with no known comorbidity who developed metforminassociated hypoglycemia that promptly resolved after stopping Met IR and initiating Met XR is presented. A thorough comparison of the pharmacokinetics of Met IR and Met XR is undertaken in the Discussion section.

\section{Case Presentation}

A 55-year-old woman with newly diagnosed T2DM was found unresponsive by her family one morning. She had been seen in the outpatient setting three days ago and initiated on Met IR $500 \mathrm{mg}$ twice daily in accordance with two consecutive fasting plasma glucose and hemoglobin A1c (HbA1c) readings of 126 and $155 \mathrm{mg} / \mathrm{dl}$, and 6.3 and $6.8 \%$, respectively, together with an insistence by the patient to "deliciously" control diabetes without an excessive cut-down on her favorite dessert. While in the ward, her daughter reported that the patient had experienced three episodes in the past two days characterized by headaches, nausea, sweating, and a feeling that she was going to pass out, despite adequate meals and adherence to Met IR as prescribed. During the most recent episode, her fingerstick glucose was $69 \mathrm{mg} / \mathrm{dl}$ and symptoms were relieved by oral fast-acting carbohydrates. Medical history included long-standing obesity only with a body mass index at present of $36 \mathrm{~kg} / \mathrm{m}^{2}$. Of note, she had no history of renal insufficiency. When last checked at the outpatient, her urinary albumin-to-creatinine ratio had been $0.22 \mathrm{mg} / \mathrm{mmol}$ (normal value: <3.4).

On presentation, she was diaphoretic and somnolent, with normal vital signs. Pupils were equal, round, and reactive to light. Further neurologic examination revealed disorientation to time and place but normal deep tendon reflexes and no focal sensory or motor deficits. The cardiopulmonary examination was normal. Her bedside point-of-care capillary blood glucose was checked and it was low, a finding confirmed by a serum blood glucose of $54 \mathrm{mg} / \mathrm{dl}$. The diagnosis prompted intravenous administration of $25 \mathrm{ml}$ of $50 \%$ dextrose. Emergent laboratory test results were unremarkable except for low blood glucose and an above-optimal lowdensity lipoprotein level (Table 1). There was no overt lactic acidosis on arterial blood gas results (pH: 7.4, $\mathrm{PaCO}_{2}: 36 \mathrm{mmHg}$, and $\left.\mathrm{HCO}_{3^{-}}: 24 \mathrm{mEq} / \mathrm{L}\right)$.

\begin{tabular}{|c|c|}
\hline Parameter & Value (reference) \\
\hline \multicolumn{2}{|l|}{ Blood count } \\
\hline Leukocyte count ( $\left.\times 10^{9} / \mathrm{L}\right)$ & $6.7(4.5-11.0)$ \\
\hline Erythrocyte count ( $\left.\times 10^{12} / L\right)$ & $4.7(3.5-5.5)$ \\
\hline Hemoglobin (g/dL) & $14.9(12-16)$ \\
\hline Hematocrit (\%) & $42(36-46)$ \\
\hline Mean corpuscular volume (fL) & $85.4(80-100)$ \\
\hline Mean corpuscular hemoglobin (pg) & $27.6(25.4-34.6)$ \\
\hline Mean corpuscular hemoglobin concentration (g/dL) & $33.3(31.5-34.5)$ \\
\hline Platelet count $\left(\times 10^{9} / \mathrm{L}\right)$ & $240(150-400)$ \\
\hline \multicolumn{2}{|l|}{ Liver function tests } \\
\hline Alanine aminotransferase (U/L) & $22(8-40)$ \\
\hline Aspartate aminotransferase (U/L) & $18(8-40)$ \\
\hline Alkaline phosphatase (U/L) & $84(45-115)$ \\
\hline Albumin (g/L) & $40(35-50)$ \\
\hline Total bilirubin ( $\mu \mathrm{mol} / \mathrm{L})$ & $4(2-17)$ \\
\hline \multicolumn{2}{|l|}{ Endocrine/miscellaneous } \\
\hline Random blood glucose (mg/dL) & 54 (hypoglycemia: $\leq 70$ ) \\
\hline
\end{tabular}




\section{Cureus}

Vitamin B12 (pmol/L)

$253(118-701)$

Thyroid-stimulating hormone (mIU/L)

$2.4(0.5-5.0)$

Renal function tests

Urea (mmol/L)

$4.4(2.5-7.1)$

Creatinine $(\mu \mathrm{mol} / \mathrm{L})$

75 (53-106)

Serum electrolytes

Sodium (mEq/L)

$138(136-145)$

Potassium (mEq/L)

$4.5(3.5-5.0)$

Chloride (mEq/L)

$102(95-105)$

Bone profile

Calcium (mmol/L)

Phosphate (mmol/L)

Coagulation profile

Prothrombin time (seconds)

$14(11-15)$

Partial thromboplastin time (seconds)

$32(25-40)$

Lipid tests

Cholesterol (mmol/L)

$4.6(3.9-6.2)$

Triglycerides (mmol/L)

$0.8(0.4-1.6)$

High-density lipoprotein (HDL) cholesterol (mmol/L)

1.2 (desirable: $>1.3$ )

Low-density lipoprotein (LDL) cholesterol (mmol/L)

3.2 (optimal: <2.6, near/above optimal: $2.6-3.4$ )

Very-low-density lipoprotein (VLDL) cholesterol (mmol/L)

$0.4(0.1-1.7)$

\section{TABLE 1: Blood investigation results}

The patient's condition improved transiently after the administration of an intravenous bolus of dextrose. However, repeat blood glucose two hours later fell to $67 \mathrm{mg} / \mathrm{dl}$ and 4 ounces of apple juice were given at this stage. Subsequently, levels began trending towards the normal range without any recurrence of hypoglycemia, reflected also by her conscious and oriented mental status, till hospital discharge. Serial measurements of blood glucose are documented in Table 2 .

Time after the morning dose of metformin immediate-release (Met IR)

$@ 0400$

$@ 0415$

@0600

$@ 0800$

@1100
Blood glucose levels (mg/dL)

$54 \rightarrow$ intravenous dextrose administered

115

$67 \rightarrow$ one 4 oz. apple juice

85

110

TABLE 2: Blood glucose levels

Though not very familiar, the temporal association of hypoglycemia with the initiation of Met IR and the fact that there were no other factors to explore caused the medication to be withheld; her therapy was transitioned to Met XR $500 \mathrm{mg}$ once a day. Over the next two weeks, a review of her self-monitored two-hour postprandial blood glucose showed levels of $125-165 \mathrm{mg} / \mathrm{dl}$. Laboratory analysis at follow-up (day 14) 
revealed the following - fasting plasma glucose: $102 \mathrm{mg} / \mathrm{dl}$, HbA1c: 5.9\%. Met XR was now titrated up by 500 $\mathrm{mg}$, i.e., $1000 \mathrm{mg}$ once a day. After three weeks of therapy with Met XR (day 21), the patient is happy because of optimal glycemic control. She has experienced no more hypoglycemic attacks since the new therapy was started. A reasonable alternative is to reduce the dose of Met IR to $250 \mathrm{mg}$ twice a day (the tablet can be cut in half), but this was not experimented on in this particular case.

\section{Discussion}

Metformin is the preferred initial medication for most patients with T2DM due to its overall efficacy and lack of hypoglycemia when used in monotherapy. Overall, the available case reports also suggest that clinically significant hypoglycemia is uncommon in metformin monotherapy and unlikely to occur abruptly in the absence of any predisposing conditions [6-11]. Indeed, there is no previous report of this phenomenon occurring merely due to Met IR, as is evident in this patient by the normal renal and liver function tests, and the absence of risk factors for hypoglycemia that include prolonged fasting, exercise, acute illness, or addition of another antidiabetic [1]. A randomized, multi-center trial compared 24 weeks of treatment with twice-daily Met IR $1000 \mathrm{mg}$ to once-daily Met XR $2000 \mathrm{mg}$ in patients with T2DM and is probably the only one that cites reports of hypoglycemia in $1.1 \%$ of patients exclusively on Met IR. Met XR was well tolerated at a single dose and there was three times less down-titration [12].

Metformin analogs are formulated with different pharmacokinetic properties, with the differences explained by a slower intestinal absorption of Met XR. Met XR provides a delayed mean time to maximum plasma concentration $\left(\mathrm{T}_{\max }\right.$ ), as compared to the $\mathrm{T}_{\max }$ provided by Met IR. The delayed $\mathrm{T}_{\max }$ occurs from four to eight hours after administration [1]. If Met XR is administered at dinner time (when dinner is normally eaten, generally between about 6 p.m. and 8 p.m.), the $\mathrm{T}_{\max }$ would occur during the time when

gluconeogenesis is expected to occur at its highest level [13]. The extent of absorption or systemic exposure is equivalent for both formulations, which is ideal especially in patients who switch to a Met XR formulation [1]. Peak plasma levels are about 20\% lower as compared to a similar dose of Met IR [1]. In this respect, three parallel studies were conducted on 78 healthy subjects. A sample size of 26 subjects per group provided $>80 \%$ statistical power to detect a difference in pharmacokinetic parameters between at least one Met XR group and Met IR. Every subject received a one-day dosing of either Met IR $1000 \mathrm{mg}$ in the fed or fasting state or Met XR $750 \mathrm{mg}$ in the fasting state. Both Met XR and Met IR displayed a similar half-life. The maximum plasma concentration $\left(\mathrm{C}_{\max }\right)$ of Met XR was much lower than that of Met IR $(\mathrm{p}<0.05)$.

Additionally, Met XR was generally well-tolerated [14].

The graph below (Figure 1) shows the plasma drug concentration-time curves of the two different oral formulations. The one represented by the gray curve shows a reduced and delayed peak level relative to the black curve, which is consistent with a sustained-release preparation. The fluctuation index $-\mathrm{C}_{\max } \operatorname{minus}$ minimum plasma concentration $\left(\mathrm{C}_{\min }\right)$ /average plasma concentration $\left(\mathrm{C}_{\text {avg }}\right)$ - is of particular interest when comparing drugs with varying release properties. The lower the fluctuation index, the more likely the $\mathrm{C}_{\max }$ is blunted while effectively extending the dosing interval, potentially allowing for up-titration to higher doses if needed for efficacy. This results in flatter concentration-time plots. There is even a benefit to having lower metformin levels in the blood during the afternoon in patients who are under concomitant therapy with at least one additional antidiabetic drug such as glyburide or its equivalents. In contrast to Met XR that does not increase endogenous insulin, a long-acting sulfonylurea, for instance, continues to stimulate pancreatic beta-cell insulin secretion even when blood glucose levels are normal (glucose-independent insulin release) and is therefore prone to causing hypoglycemia $[1,15]$. 


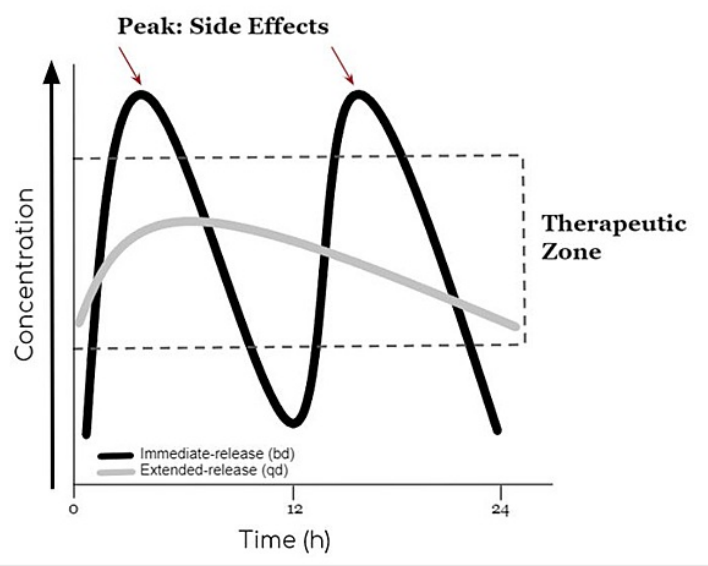

FIGURE 1: Simulated pharmacokinetic comparison of formulations of the same drug to compare immediate- and extended-release properties

bd: twice daily; qd: once daily

Some degree of research has been performed in the area of controlled-release compositions that employ antihyperglycemic drugs. The value of Met XR is further indicated by the results of a randomized, doubleblind, active-controlled, six-month clinical trial among adult patients with drug-naive T2DM and HbA1c levels $>7.0 \%$ and $<8.5 \%$ [3]. Metformin was given in a randomized sequence at the maximum tolerated dose as Met IR in one group and as Met XR in the other. The efficacy endpoint was glycemic control as determined by the reduction in $\mathrm{HbA1C}$, fasting plasma glucose, and postprandial plasma glucose levels after six months. Accordingly, insulin resistance was also measured. Met XR produced not only a statistically significant and sustained reduction in these measures of glycemic control compared with baseline $(p<0.01)$ but also a more efficacious lowering of levels than Met IR $(\mathrm{p}<0.05)$. Secondly, the reduction in insulin resistance was consistent with these changes ( $\mathrm{p}<0.01 \mathrm{vs}$. baseline, $\mathrm{p}<0.05 \mathrm{vs}$. Met IR). Of note, there was no significant increase in adverse events with an average $1000 \pm 500 \mathrm{mg} /$ day dose of Met XR, even with improved efficacy at this dose. Although there were no cases of hypoglycemia in either group, the overall incidence of gastrointestinal side effects was lower among those who received Met XR. While there may be other explanations for the improved outcome on Met XR, including enhanced compliance due to less frequent dosing, minimizing fluctuations in serum drug levels or continued glycemic control is likely to have played an important role [3]. In the present case too, Met XR achieved "therapeutically effective reduction", which signifies that plasma glucose levels were reduced by an effective amount compared to a Met IR reference standard when the dosage form was orally administered to the patient on a once-daily basis.

Further contemplated for the purpose of the present case is that Met IR persisted at its peak plasma levels for approximately two hours, although this claim is directed to the pharmacokinetic parameters of an individual patient. A limitation of this study is that serum metformin levels were not determined. The Naranjo algorithm, a tool used to discern an adverse drug reaction, was used retrospectively to analyze this case presentation. According to the algorithm, this case showed a score of 7 , which coincides with a "probable" diagnosis of adverse drug reaction. This is supported by clinical improvement and resolution of hypoglycemic symptoms with the discontinuation of Met IR. This patient had symptomatic hypoglycemia, confirmed by the Whipple triad [16], and is by definition "severe hypoglycemia" or "documented symptomatic hypoglycemia" [17].

\section{Conclusions}

In accordance with the dosage forms of the present case, it has been determined that this patient suffering from T2DM achieved improved results (e.g., effectively lowered blood glucose levels, lower risk of hypoglycemia) with Met XR than Met IR administered according to popular protocols, e.g., on a twice-a-day basis. Another clear advantage is a reduction in the frequency of administration with Met XR. All of these findings suggest that Met XR may improve the quality of therapy and the safety profile relative to a conventional dosage form in patients with T2DM.

\section{Additional Information}

\section{Disclosures}

Human subjects: Consent was obtained or waived by all participants in this study. Conflicts of interest: In compliance with the ICMJE uniform disclosure form, all authors declare the following: Payment/services info: All authors have declared that no financial support was received from any organization for the 
submitted work. Financial relationships: All authors have declared that they have no financial relationships at present or within the previous three years with any organizations that might have an interest in the submitted work. Other relationships: All authors have declared that there are no other relationships or activities that could appear to have influenced the submitted work.

\section{Acknowledgements}

Armed Forces Institute of Pathology (AFIP), for its prompt reporting of laboratory results.

\section{References}

1. GLUCOPHAGE ${ }^{\circledR}$ (metformin hydrochloride tablets) GLUCOPHAGE ${ }^{\circledR}$ XR (metformin hydrochloride extended-release tablets). (2006). Accessed: June 9, 2021: https://www.accessdata.fda.gov/drugsatfda_docs/label/2006/020357s030,021202s015lbl.pdf.

2. Madiraju AK, Erion DM, Rahimi Y, et al.: Metformin suppresses gluconeogenesis by inhibiting mitochondrial glycerophosphate dehydrogenase. Nature. 2014, 510:542-6. 10.1038/nature13270

3. Derosa G, D'Angelo A, Romano D, Maffioli P: Effects of metformin extended release compared to immediate release formula on glycemic control and glycemic variability in patients with type 2 diabetes. Drug Des Devel Ther. 2017, 11:1481-8. 10.2147/DDDT.S131670

4. Extended release drugs: are they right for you? . (2017). Accessed: June 9, 2021: https://www.goodrx.com/blog/extended-release-drugs-are-they-right-for-you/.

5. Jabbour S, Ziring B: Advantages of extended-release metformin in patients with type 2 diabetes mellitus Postgrad Med. 2011, 123:15-23. 10.3810/pgm.2011.01.2241

6. Yang PW, Lin KH, Lo SH, Wang LM, Lin HD: Successful treatment of severe lactic acidosis caused by a suicide attempt with a metformin overdose. Kaohsiung J Med Sci. 2009, 25:93-7. 10.1016/S1607551X(09)70047-6

7. Rathnapala A, Matthias T, Jayasinghe S: Severe lactic acidosis and acute renal failure following ingestion of metformin and kerosene oil: a case report. J Med Case Rep. 2012, 6:18. 10.1186/1752-1947-6-18

8. Al-Abri SA, Hayashi S, Thoren KL, Olson KR: Metformin overdose-induced hypoglycemia in the absence of other antidiabetic drugs. Clin Toxicol (Phila). 2013, 51:444-7. 10.3109/15563650.2013.784774

9. Zitzmann S, Reimann IR, Schmechel H: Severe hypoglycemia in an elderly patient treated with metformin . Int J Clin Pharmacol Ther. 2002, 40:108-10. 10.5414/cpp40108

10. Gjedde S, Christiansen A, Pedersen SB, Rungby J: Survival following a metformin overdose of $63 \mathrm{~g}$ : a case report. Pharmacol Toxicol. 2003, 93:98-9. 10.1034/j.1600-0773.2003.930207.x

11. Aldobeaban S, Mzahim B, Alshehri AA: Recurrent hypoglycemia secondary to metformin toxicity in the absence of co-ingestions: a case report. J Med Case Rep. 2018, 12:223. 10.1186/s13256-018-1758-0

12. Aggarwal N, Singla A, Mathieu C, et al.: Metformin extended-release versus immediate-release: an international, randomized, double-blind, head-to-head trial in pharmacotherapy-naïve patients with type 2 diabetes. Diabetes Obes Metab. 2018, 20:463-7. 10.1111/dom.13104

13. Magnusson I, Rothman DL, Katz LD, Shulman RG, Shulman GI: Increased rate of gluconeogenesis in type II diabetes mellitus. A 13C nuclear magnetic resonance study. J Clin Invest. 1992, 90:1323-7. 10.1172/JCI115997

14. Idkaidek N, Arafat T, Melhim M, Alawneh J, Hakooz N: Metformin IR versus XR pharmacokinetics in humans. J Bioequiv Biovailab. 2011, 3:233-5. 10.4172/jbb.1000092

15. Sola D, Rossi L, Schianca GP, et al.: Sulfonylureas and their use in clinical practice . Arch Med Sci. 2015, 11:840-8. 10.5114/aoms.2015.53304

16. Mokán M: Hypoglycaemia (Article in Slovak). Vnitr Lek. 2008, 54:387-94.

17. Seaquist ER, Anderson J, Childs B, et al.: Hypoglycemia and diabetes: a report of a workgroup of the American Diabetes Association and the Endocrine Society. Diabetes Care. 2013, 36:1384-95. 10.2337/dc122480 